\title{
Application of Solar Electric Propulsion to a Comet Surface Sample Return Mission
}

\author{
Mike Cupples ${ }^{\dagger}$ \\ Science Applications International Corporation \\ Huntsville, Alabama 35806 \\ Victoria Coverstone* and Byoungsam Woo** \\ University of Illinois at Urbana-Champaign, \\ Urbana, Illinois 61801
}

Current NSTAR (planned for the Discovery Mission: Dawn) and NASA's Evolutionary Xenon Thruster based propulsion systems were compared for a comet surface sample return mission to Tempel 1 . Mission and systems analyses were conducted over a range of array power for each propulsion system with an array of $12 \mathrm{~kW}$ EOL at 1 AU chosen for a baseline. Engine configurations investigated for NSTAR included 4 operational engines with 1 spare and 5 operational engines with 1 spare. The NEXT configuration investigated included 2 operational engines plus 1 spare, with performance estimated for high thrust and high Isp throttling modes. Figures of merit for this comparison include Solar Electric Propulsion dry mass, average engine throughput, and net non-propulsion payload returned to Earth flyby.

\section{Introduction}

With the success of the Deep Space 1 mission ${ }^{1,2}$, the potential of Solar Electric Propulsion Systems (SEPS) was clearly demonstrated, making SEPS a strong candidate for various interplanetary missions. The long-duration, highefficiency operation of SEPS allows new ways to explore the inner and outer solar system, and enables missions that can be difficult and expensive to reach with chemical propulsion systems. NASA has undertaken an ion propulsion system development program to create a new ion propulsion system based on NASA's Evolutionary Xenon Thruster $^{3}$ (NEXT). This thruster development is being developed under of the NEXT Generation Electric Propulsion (NGEP) Technology Area ${ }^{4}$ under the auspices of the In-Space Propulsion Technology Projects at Marshal Space Flight Center (MSFC). The NEXT based propulsion system has a higher maximum design power

\footnotetext{
† Systems Engineer, In-Space Technology Assessment Lead, Science Applications International Corporation, 6725 Odyssey Drive, Huntsville, AL 35806

* Associate Professor, Department of Aeronautical and Astronautical Engineering, 306 Talbot Laboratory, 104 S. Wright Street, Urbana, IL 61801, Member AAS,

Associate Fellow AIAA

** Graduate Student, Department of Aeronautical and Astronautical Engincering, 306 Talbot Laboratory, 104 S. Wright Street, Urbana, IL 6180 



\section{Mission Assumptions.}

A summary of mission assumptions are as follows:

1) Mission Type - Comet surface sample return

2) Target - comet Tempel 1

3) Launch Date - 2008 Earth Departure

4) Stay Time @ Target - 60 days

The payload is defined in this study as $50 \mathrm{~kg}$ of mass that remains at the comet and the net non-propulsive mass that is returned to Earth. This net return mass consists of the comet sample, comet sample return capsule with concomitant mechanisms, and the remaining spacecraft mass (e.g. subsystem including navigation instruments, communication instruments, science instruments, structure, power, and thermal).

Figure 1 depicts orbits of the Earth and comet Tempel 1 orbits around the sun. The primary objective of the mission is for the spacecraft to rendezvous with Tempel 1, collect a sample, and return the sample to Earth. A launch vehicle will place the SEPS spacecraft on an optimal Earth escape trajectory, and the SEPS propulsion will provide an optimal transfer to rendezvous with Tempel 1 and provide return propulsion for a flyby of Earth at sample delivery.

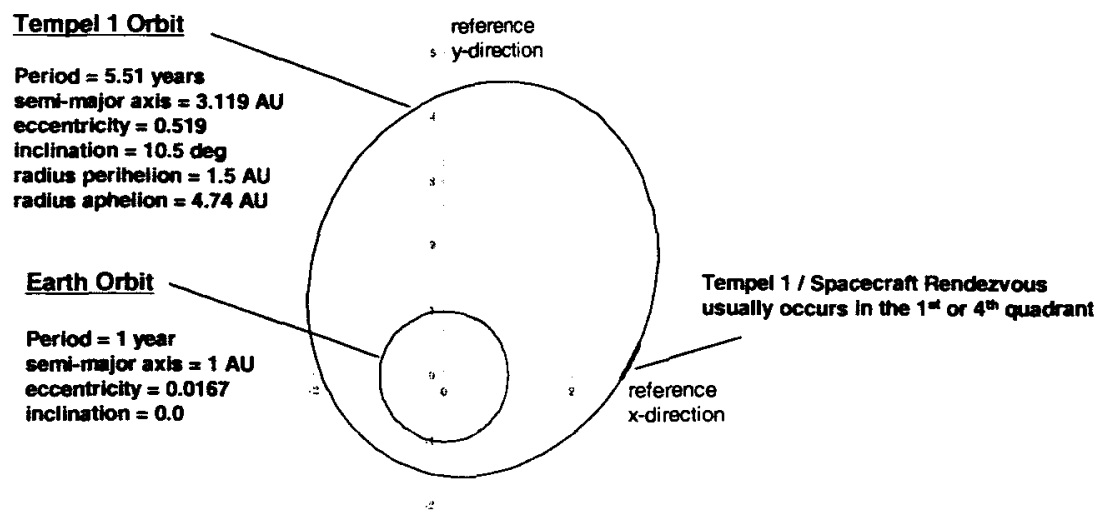

Figure 1 Earth and Tempel 1 Solar System Orbit Depiction 


\section{Systems Assumptions}

Launch Vehicle. The launch vehicle ${ }^{12}$ (LV) chosen for this investigation was the Delta IV 4040 . This LV is the smallest Delta medium class LV, and matches well the low cost objective of a New Frontiers class sample return mission. The LV modeling details that were included in the trajectory optimization process are provided below in Figure 2. This study assumed a LV margin of $0 \%$.
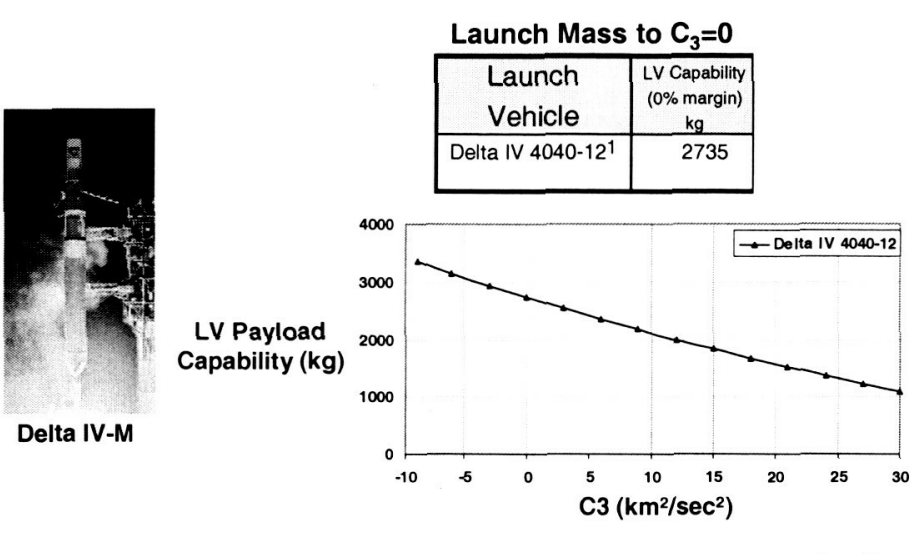

Figure 2 LV performance model

Solar Electric Propulsion. Detailed SEP system model were developed and applied to the analyses conducted in this study. The system models for each case of this study include power and power conversion/distribution, thrusters, power processing units, propellant management and tank, structures, and thermal control. SEP system models are either physics based models or models derived from scaling of actual spacecraft. For example the SEPS structure model was derived from a spacecraft database including Cassini, Galileo, Mars Global Surveyor, Near, Odyssey, and Mars Climate Orbiter. A detailed summary of the system models is provided in Figure 3. 


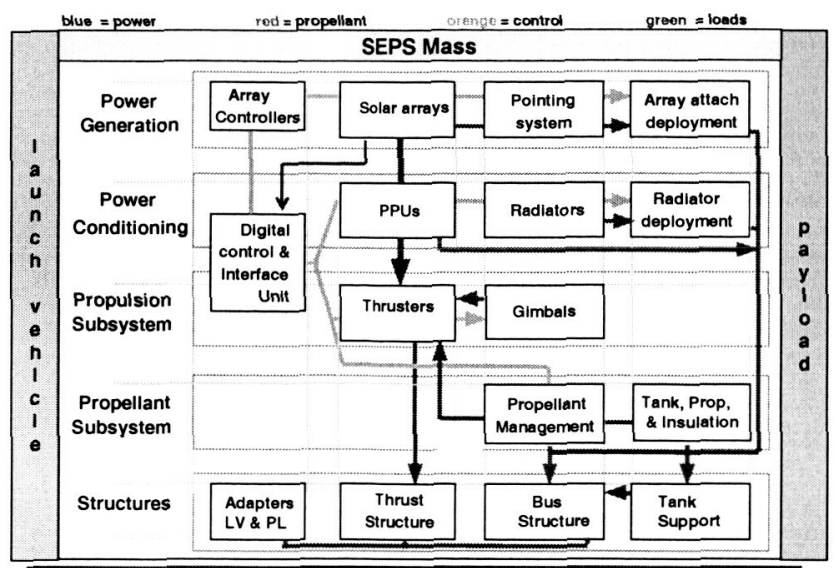

SEP system models are detailed and incorporate expert based algorithms

Figure 3 SEP System Model Depiction

The propulsion configurations investigated are shown in Figure 4 below.

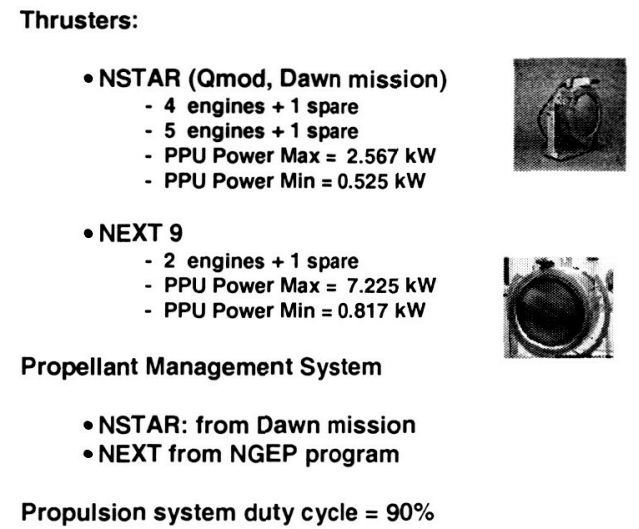

Figure 4 Thruster, PPU, and PMS systems analysis assumptions

The NSTAR thruster, sometimes termed the Qmod-NSTAR, is based on Molybdenum gridded ion optics. The NEXT thruster is based on Molybdenum gridded ion optics as well.

System Power. The solar arrays were assumed as the only source of propulsion power for the system. The array power model employed in the trajectory optimization task is multi-junction GeAs array technology ${ }^{13}$ with Ultraflex light weight array design ${ }^{14}$. The performance of this solar array technology is slightly below the $1 / \mathrm{r}^{2}$ solar insolation. 
The Ultra-Flex array concept is depicted in the left side of Figure 5. Other necessary.power system modeling assumptions are shown in Figure 5 as well.

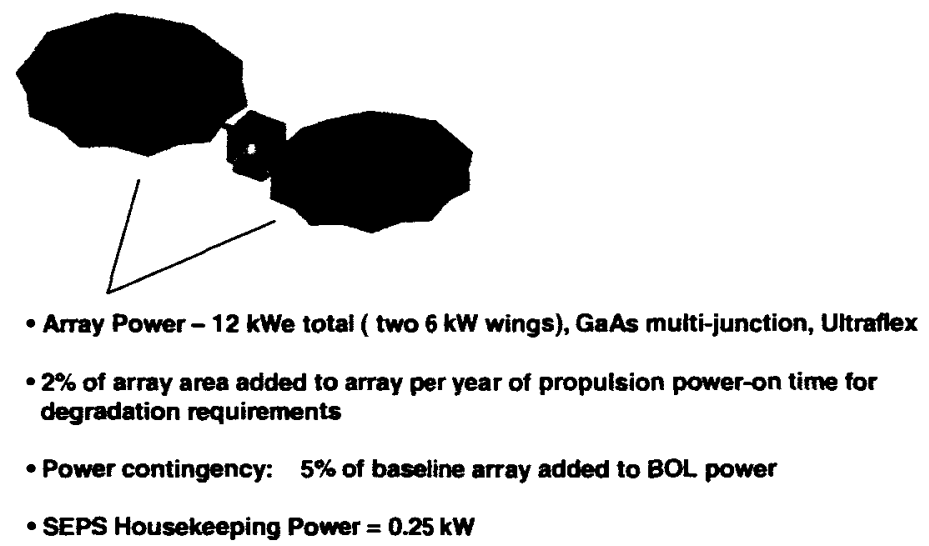

Figure 5 Solar array system modeling assumptions

Other Assumptions. Several other systems assumptions including Xe propellant contingency, systems dry mass contingency, redundancy, and ACS are summarized in the summary in Table 1.

Table 1 Miscellaneous systems analysis assumptions

\begin{tabular}{|l|l|}
\hline Contingency & $\begin{array}{r}\text { Propellant: } \\
10 \% \text { of Xe deterministic, NSTAR (Dawn Mission Assumption) } \\
8.6 \% \text { of Xe deterministic, NEXT (NGEP Program Assumption) }\end{array}$ \\
& Dry Mass: $30 \%$ of current best estimate \\
\hline Redundancy & One extra ion system ( thruster, PPU, propellant distribution string, and DCIU) \\
\hline ACS & ACS provided by IPS during low thrust burn \\
& ACS provided by RCS during periods when IPS is not active \\
\hline
\end{tabular}

In this phase of analysis, work to delineate the spacecraft mass with the concomitant instruments and mechanisms to make a successful comet sample return is incomplete. Further spacecraft definition is expected to be performed in the near future to address this need. 


\section{Trajectory Optimization}

Trajectory optimization with variable thrust and thrust direction has been previously investigated ${ }^{15,16,17}$. The solution typically requires the optimization a number of parameters. For the case investigated, the final delivered mass to Earth return is maximized.

Trajectory optimization was performed using Solar Electric Propulsion Trajectory Optimization Program $(\text { SEPTOP })^{18}$. SEPTOP is a two-body, Sun-centered, low-thrust trajectory optimization program for preliminary mission feasibility studies that provides relatively accurate performance estimates. The program determines a numerical solution to a two point boundary value problem that satisfies intermediate boundary constraints. In SEPTOP, the user estimates initial conditions, and then uses a shooting method to integrate the trajectory from an initial time to final time. SEPTOP computes an error at the final time and uses it to correct the estimate of the initial conditions. This process is repeated until the error becomes smaller than the prescribed tolerance. The required inputs are Time-of-flight, nominal Earth departure, array power at Earth departure, maximum power into Power Processing Unit (PPU), intermediate rendezvous body (in this case comet Tempel 1), and LV specifications. SEPTOP can model variable thrust and mass flow rate as a function of power into the PPU. The power generated from a solar array is modeled as a function of the spacecraft's distance from the Sun. Thruster and solar array models are therefore also required as inputs.

\section{Performance Comparison}

Figure 6 shows a typical Tempel 1 roundtrip comet sample return trajectory. This trajectory, based on an optimized NEXT high thrust throttling mode, required approximately 8 years in total transfer time. The salient trajectory features include the following:

1) LV assisted Earth departure to a $\mathrm{C} 3$ of $\sim 14.3 \mathrm{~km} \wedge 2 / \mathrm{sec}^{\wedge} 2$

2) SEPS assisted transfer to rendezvous with Tempel 1

3) 60 day stay at Tempel 1 , during which a sample is taken from the comet and returned to the spacecraft

4) SEPS assisted return to an Earth flyby to allow sample return to Earth. 


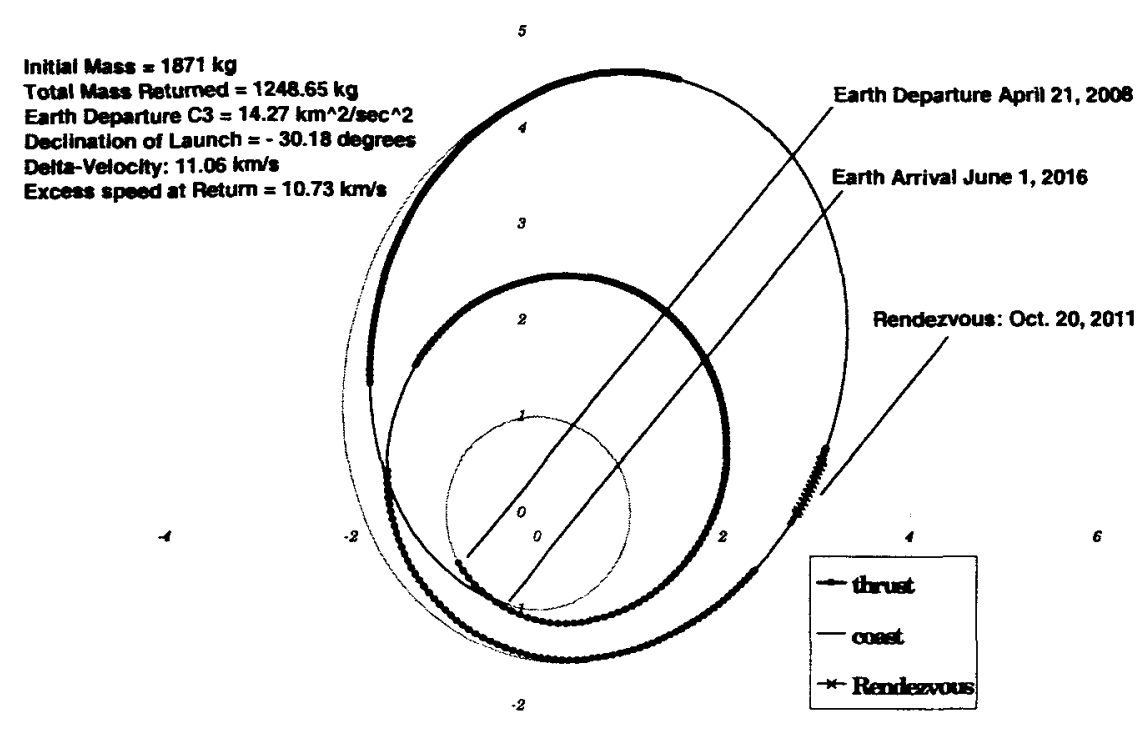

Figure 6 Typical comet surface sample return trajectory

Derived results for this example trajectory are as follows: SEPS wet mass $=1249 \mathrm{~kg}$, SEPS dry mass $=623 \mathrm{~kg}$, total payload (not including the $50 \mathrm{~kg}$ remaining at the comet) $=622 \mathrm{~kg}$ and the average engine throughput $=308 \mathrm{~kg}$.

Variation in Performance Due to Array Power and Operational Thrusters. Two case studies of payload vs array power are depicted in Figures 7 and 8 . For the first case, Figure 7 shows the result of varying the number of operational propulsion strings (thruster, ppu, and propellant distribution and control). Given that five thrusters at a maximum power each of $2.567 \mathrm{~kW}$ implies a maximum power usage of over $12.8 \mathrm{~kW}$ at $1 \mathrm{AU}$, the $5+1$ configuration allows the system to utilize all of the available array power (minus housekeeping) at $1 \mathrm{AU}$. Four thrusters at a maximum power each of $2.567 \mathrm{~kW}$ implies a maximum power usage of less than $10.3 \mathrm{~kW}$ at $1 \mathrm{AU}$ implies that the $4+1$ configuration does not permit the full array power to be utilized at $1 \mathrm{AU}$. Yet as Figure 7 shows, the NSTAR $4+1$ configuration performed modestly better than the $5+1$ configuration due to the overpowering increase in dry mass for the $5+1$ case. 


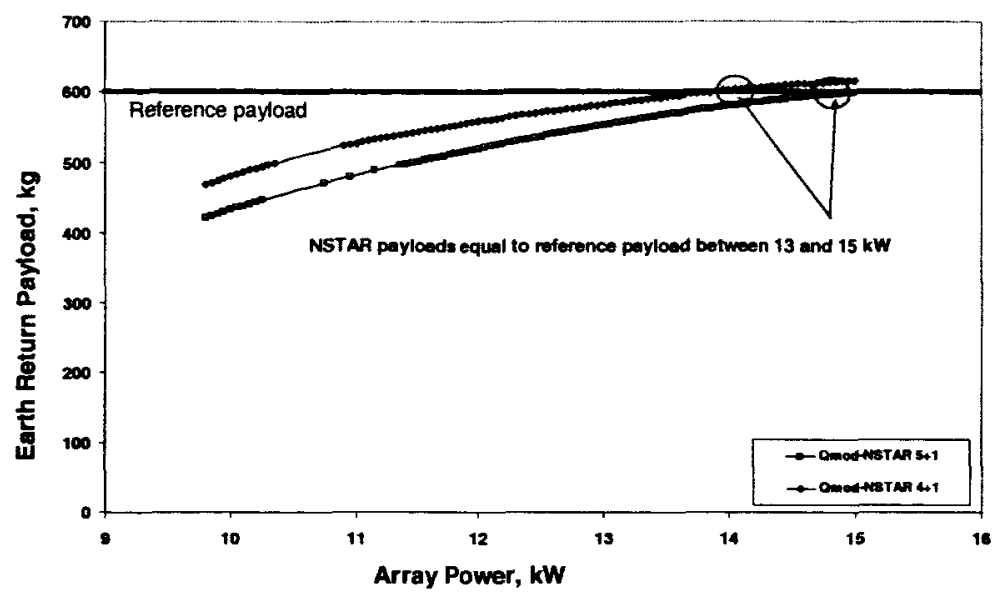

Figure 7 NSTAR payload for a range of array power and engine configuration

The second case study investigated the difference in performance between the NEXT high thrust and high Isp throttling modes, with further comparison to the NSTAR thruster. Notice from Figure 8 that the NSTAR and NEXT throttling level cross well before $12 \mathrm{~kW}$, but $12 \mathrm{~kW}$ falls on the curves well above the higher second derivative area of the curves below approximately $10 \mathrm{~kW}$. Hence the choice of $12 \mathrm{~kW}$ as a baseline power level to perform much of the analyses of this study. In general, the NEXT thruster performed better than NSTAR above a power level of $\sim 10 \mathrm{~kW}$. A comparison of NEXT high thrust throttling and high Isp throttling in Figure 8 indicated that the high thrust throttling mode provides a small decrease in required power for a given payload. At the $12 \mathrm{~kW}$ baseline array power, the high thrust throttling yields a net increase of $\sim 15 \mathrm{~kg}$ over high Isp throttling.

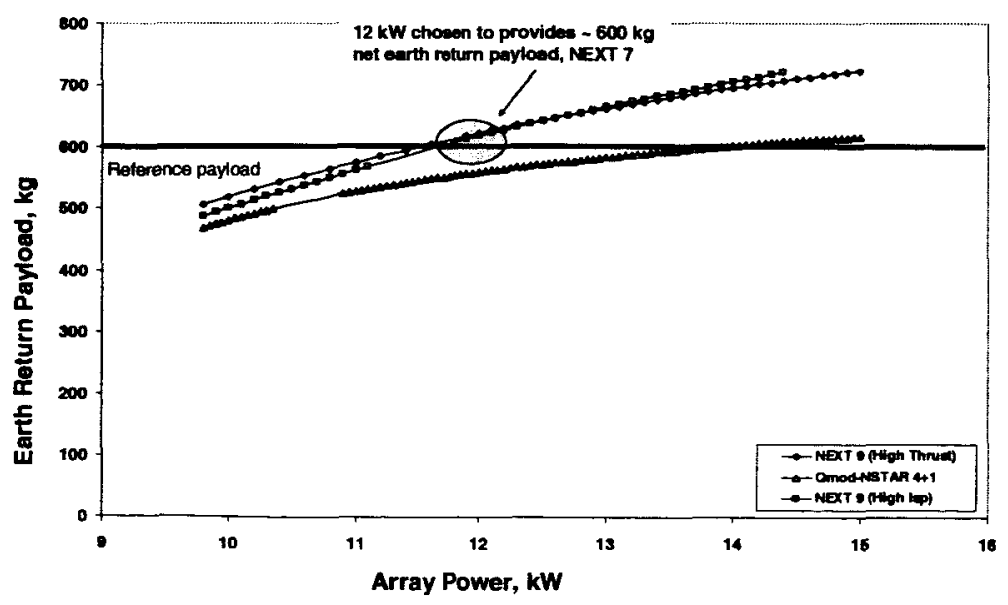

Figure 8 NEXT and NSTAR payload comparison over a range of array power 
Detailed Comparison of Payload Delivery Performance at Baseline Array Power. A comparison of NSTAR and NEXT was performed at a power level of $12 \mathrm{~kW} .12 \mathrm{~kW}$ was chosen as a baseline power to perform this analysis for two reasons: the first is that this power level corresponds to a little more than $600 \mathrm{~kg}$ of Earth return payload for NEXT and is near a cross-over in payload between high thrust throttling and high Isp throttling; the second reason for the $12 \mathrm{~kW}$ baseline array power is that a NEXT phase 1 study was performed at $12 \mathrm{~kW}$, thus allowing the study herein to relate to earlier studies with some continuity. The previous mentioned power level sweep indicated that above the power level of $\sim 10 \mathrm{~kW}$, NEXT provides an increasing advantage in payload mass as the power level increases.

An overall stack mass comparison is shown in Figure 9. The case of NSTAR with a $4+1$ engine configuration delivers over $\sim 559 \mathrm{~kg}$ to Earth, as compared to 520 for the $5+1$ engine configuration. The NEXT cases using the high thrust throttling mode deliver about $622 \mathrm{~kg}$ to Earth return, and the cases utilizing the high Isp throttling mode deliver approximately $618 \mathrm{~kg}$ to Earth return. Thus, for the Tempel 1 CSSR mission, both NEXT cases showed significant improvement over NSTAR in payload delivery capability. Also this NEXT payload delivery was realized with a $2+1$ engine configuration that may imply significant cost and complexity benefits over the $4+1$ NSTAR configuration.

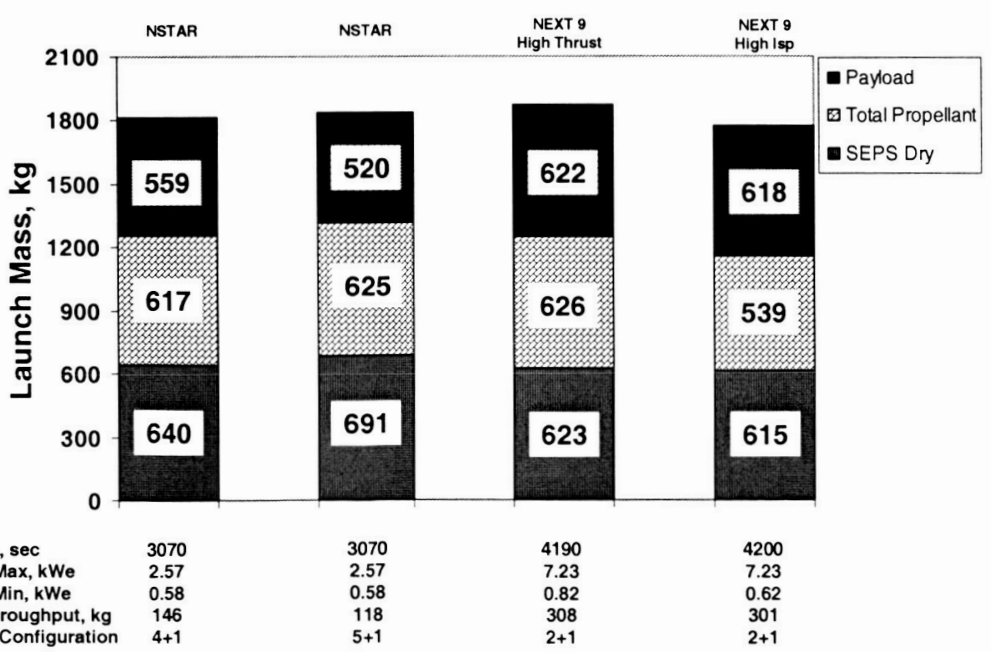

Figure 9 Comparison of mission stack mass for NEXT and NSTAR 
In addition to the payload FOM, SEPS dry mass and average engine throughput is shown in Figure 9. In general, the NEXT SEPS is less massive than NSTAR SEPS because of the lower number of propulsive strings required to perform the mission. In all NSTAR cases and in the NEXT high Isp case, the average throughput is less than the engine design requirements of $150 \mathrm{~kg}$ and $300 \mathrm{~kg}$ respectively. The NEXT high thrust throttling mode case exceeded the design requirement by approximately $8 \mathrm{~kg}$.

Multi-Engine Operation and Power Available to IPS Time Histories. Figures 10, 11, and 12 show engine on-off time histories with their corresponding ion propulsion system power levels for each of the cases identified in Figure 9 above. For each of the cases, the array power level is $12 \mathrm{~kW}$ at $1 \mathrm{AU}$, and the transfer time is approximately 8.1 years. These graphs provide power profiles as a function of mission time, and facilitate a determination of how well the ion propulsion system is utilizing the power during the course of the mission.
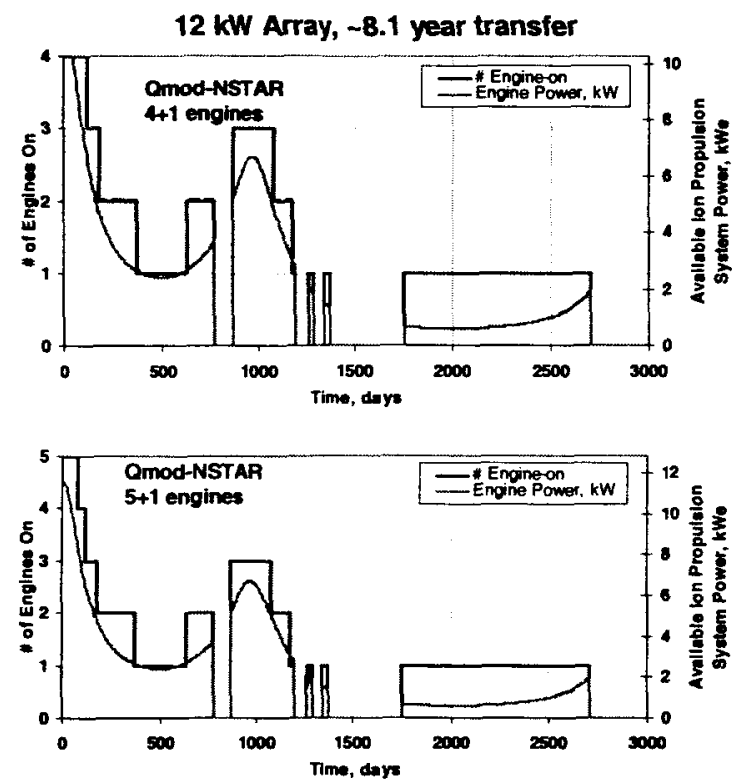

Figure 10 NSTAR engine operation and propulsion system power time history

NSTAR 4+1 and 5+1 engine configurations are compared in Figure 10. For most of the time during the mission, three or less thrusters are required, and for only a short time during the beginning of the mission, 4 or 5 thrusters are in operation. Thus, Figure 11 provides some corroborating evidence for the better performance seen earlier for the $4+1$ configuration, given that for the $5+1$ configuration the very small increase in overall power utilization 
improvement is overcome by the relatively large increase in system dry mass. From Figure 10, it can also be surmised that 4 thrusters (or 5 thrusters, depending on the case) are needed for only a short time during the beginning of the transfer. Thus, it would be possible to eliminate the spare thruster (along with the related mass) for the mission, but the mission risk would increase significantly. A similar argument could be made for the NEXT $2+1$ configuration shown in Figures 11 and 12, but mission risk would increase as well.

A comparison of the NSTAR $4+1$ configuration with the NEXT high thrust and high Isp throttling modes are provided in the following graphs, Figures 11 and 12.
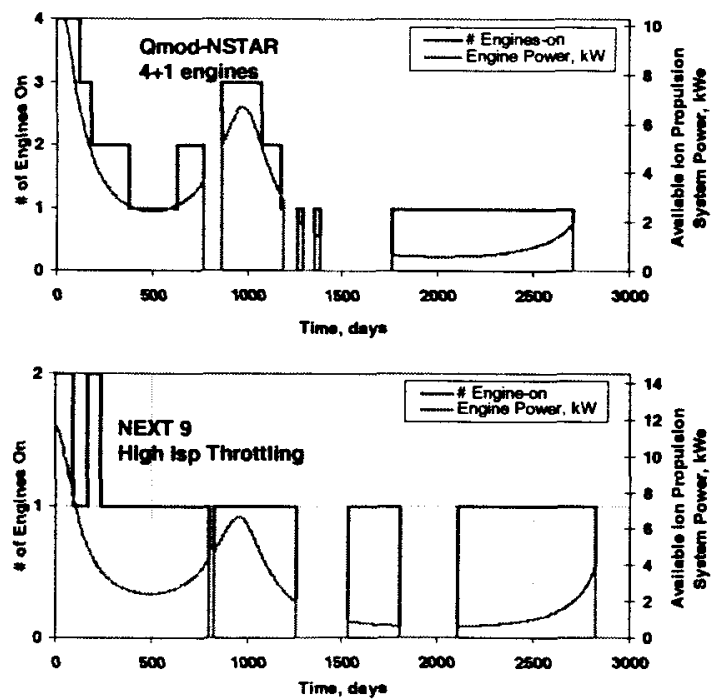

Figure 11 Engine and power time history comparison for NEXT high Isp Throttling and NSTAR 

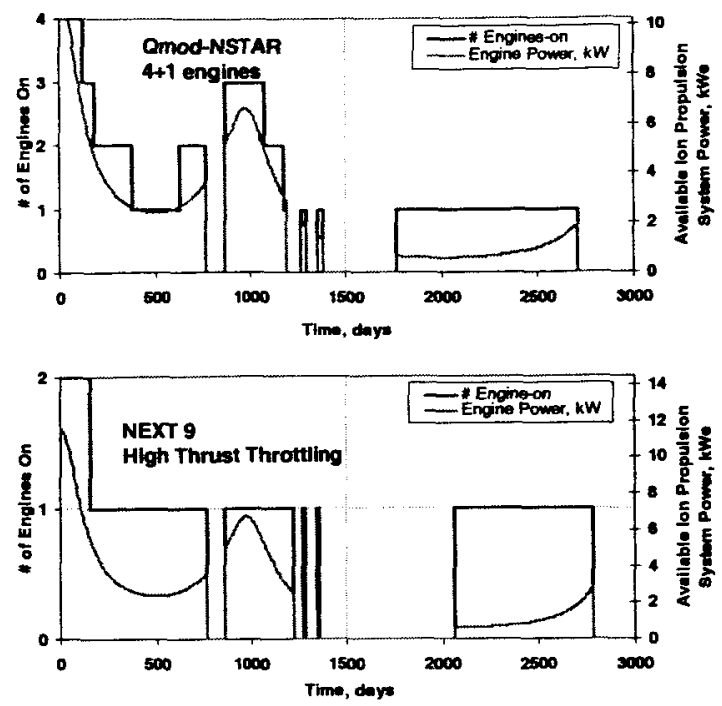

Figure 12 Engine and power time history comparison for NEXT high thrust throttling and NSTAR

Even though, as seen in the previous results of Figure 10 above, the performance of NEXT high thrust throttling is somewhat better than NEXT high Isp throttling, but this difference in NEXT engine/power profiles show only very slight differences. In general, the NEXT engine performs with one engine on for the majority of the mission, with two engines on for only approximately the first 100 days. Also note that due to the power minimum for NEXT being at the level of $0.82 \mathrm{~kW}$, there is a characteristic drop-out in engine operation between 1600 and 2400 days because the solar insolation can not provide the minimum thruster power. The NSTAR thruster, however, with a lower thruster power minimum can operate over the entire trajectory. This ability to operate at high AU provides some advantage in performance for the NSTAR SEPS, but this advantage is outweighed by the increase in dry mass due to the increased number of thrusters required (NSTAR $4+1$ vs NEXT 2+1) for the $12 \mathrm{~kW}$ power level.

\section{Summary and Conclusions}

NSTAR and NEXT based propulsion systems were compared for a comet surface sample return mission to Tempel 1. A range of array power was investigated for each propulsion system, and $12 \mathrm{~kW}$ EOL at $1 \mathrm{AU}$ was chosen as a baseline array power for this analysis. Engines configurations investigated for NSTAR included $4+1$ and $5+1$ engines, and the NEXT configuration only included a $2+1$ case. For NSTAR, it was found that the $4+1$ engine configuration provided modest performance advantage over the $5+1$ configuration. The NEXT $2+1$ configuration was then compared to the NSTAR 4+1, with a small performance advantage going to the NEXT thruster. It should be noted, however, that the NEXT $2+1$ SEPS configuration is lighter in dry mass and less complex in hardware 
implementation, resulting in an expected significantly less costly SEPS vehicle than the NSTAR 4+1 SEPS vehicle. In both the NEXT and NSTAR cases, a power/engine-on time history showed smooth variation in thruster on-off sequences following the available power to the IPS. It was found for NSTAR that 4 thrusters are needed for only a short time during the beginning of the transfer. Thus, it would be possible to eliminate the spare thruster (along with the related mass) for the mission, but the mission risk would increase significantly. A similar argument could be made for the NEXT $2+1$ configuration, but mission risk would increase as well. Finally, engine throughput for NEXT and NSTAR are within the accepted assumed limits of $300 \mathrm{~kg}$ for NEXT and $150 \mathrm{~kg}$ for NSTAR for all cases investigated except for the NEXT high thrust throttling case.

\section{Acknowledgements}

The work described in this paper was performed by Science Applications International Corporation (SAIC) under contract with the NASA Marshall Space Flight Center (MSFC). Special thanks go to Les Johnson, manager of NASA MSFC In-Space Propulsion Technology Investment Projects, and Randy Baggett, manager of NASA MSFC Next Generation Electric Propulsion Technology Area, for providing encouragement and direction for this work.

\section{References}

\footnotetext{
${ }^{1}$ Rayman, M. D., and Williams, S. N., "Design of the First Interplanetary Solar Electric Propulsion Mission," Journal of Spacecraft and Rockets, Vol. 39, No. 4, 2002, pp. 589-595

${ }^{2}$ Rayman, M. D., Chadbourne, P. A., Culwell, J. S., and Williams, S. N., "Mission Design for Deep Space 1: A Low-Thrust Technology Validation Mission," International Academy of Astronautics, Paper L98-0502, April 1998.

${ }^{3}$ Michael J. Patterson, John E. Foster, Thomas W. Haag, and George C. Soulas, "Next: NASA'S Evolutionary Xenon Thruster Development Status," 39 $9^{\text {th }}$ Joint Propulsion Conference, AIAA 2003-4862, Huntsville, AL, July 2003.

${ }^{4}$ Johnson, C.L., Alexander, L., Baggett, R.M., Bonometti, J.A., Herrmann, M., James, B.F., Montgomery, S.E., "NASA's In-Space Propulsion Technology Program: Overview and Status," 2004 JANNAF, Las Vegas, NV, May 2004.

${ }^{5}$ Tan, G.H., Sims, J.A., "Mission Design for the Deep Space 4/Champollion Comet sample Return Mission," AAS 98-187, AAS/AIAA Space Flight Mechanics Meeting, Monterey, CA, February, 1998.

${ }^{6}$ Sims, J.A., "Trajectories to Comets Using Solar Electric Propulsion," AAS 2000-134, AAS/AIAA Space Flight Mechanics Meeting, Clearwater, FL., January 2000.

${ }^{7}$ Cupples, M.L., Green, S.H., Coverstone, V., “Factors Influencing Solar Electric Payload Delivery to Outer Planet Missions," 2003 AAS/AIAA Space Flight Mechanics Conference, AAS 03-123.
} 
${ }^{8}$ Cupples, M., Green, S., Donahue, B., Coverstone, V., "Solar Electric and Chemical Propulsion for a Titan Mission," 39 ${ }^{\text {th }}$ Joint Propulsion Conference, AIAA 2003-4728, July 2003.

${ }^{9}$ Byoungsam, W., Coverstone, V., Hartmann, J., Cupples, M., "Trajectory and Systems Analysis for Outer Planet Solar Electric Propulsion Missions," Journal of Spacecraft and Rockets, pending publication, 2004.

${ }^{10}$ Oh, David., Sims, J., Benson, S., Gefert, L., Witzberger, K., Cupples, M., "Deep Space Applications of the NEXT Thruster," 40 4 th Joint Propulsion Conference, AIAA 2004- , Ft. Lauderdale, FL., July 2004.

${ }^{11}$ http://centauri.larc.nasa.gov/newfrontiers/

${ }^{12}$ http://elvperf.ksc.nasa.gov

${ }^{13}$ T. Kerslake, "Photovoltaic Array Performance during an Earth-to-Jupiter Heliocentric Transfer," NASA Glenn Research Center, PS-496, Aug. 2000.

${ }^{14}$ http://www.aec-able.com/arrays/ableultraflex.html

${ }^{15}$ Sauer, C. G., "Optimization of Multiple Target Electric Propulsion Trajectories," AIAA $11^{\text {th }}$ Aerospace Sciences Meeting, AIAA Paper 73-205, Washington, DC, Jan. 1973

${ }^{16}$ Melbourne, W. G., Richardson, D. E., and Sauer, C. G., "Interplanetary Trajectory Optimization with PowerLimited Propulsion Systems," Jet Propulsion Laboratory, TR No. 32-173, Pasadena, CA, 1962.

${ }^{17}$ Williams, S. N., and Coverstone, V. L., "Mars Missions Using Solar Electric Propulsion," Journal of Spacecraft and Rockets, Vol. 37, No. 1, 2000, pp. 71-77.

${ }^{18}$ Williams, S. N., "An Introduction to the use of VARITOP A general purpose low-thrust trajectory optimization program,” Jet Propulsion Laboratory, JPL D-1 1475, Pasadena, CA, 1994. 